\title{
A Tool Supporting Model Based User Interface Design in 3D Virtual Environments
}

\author{
Joan De Boeck, Chris Raymaekers, Karin Coninx \\ Hasselt University, Expertise Centre for Digital Media and Transnationale Universiteit Limburg \\ Wetenschapspark 2, BE-3590 Diepenbeek, Belgium \\ \{joan.deboeck, chris.raymaekers, karin.coninx\}@uhasselt.be
}

\begin{abstract}
Keywords: Multimodal Interaction, Interaction Technique, Interactive Virtual Environment, Model-Based Design
Abstract: Although interactive virtual environments (IVE) have the capability to offer intuitive and easy to use interfaces, their creation is often a long and expensive process, in which specialists play a key role. The VR-DeMo approach investigates how the use of high-level specifications may help to overcome this problem: instead of coding an IVE using a low level programming language, high-level models are used. As such a model-based process combines a series of models containing a mixture of manual and automatic processes. The usefulness of a model based process relies on available tool support. Supporting the VR-DeMo process, this paper introduces CoGenIVE. This tool has been used in order to develop a series of demonstrators, all based on reallife cases in different domains. From this experience, the VR-DeMo approach and more particular CoGenIVE have proven to be useful to develop interactive virtual environments using high-level specifications.
\end{abstract}

\section{Introduction}

Interactive Virtual environments (IVE) are computer applications that try to create the effect of a 3D world in which a user can interact as easily and intuitively as possible, preferably using multiple senses such as the hearing, the sight and touch. In contrast with standard desktop applications, however, the development of such an environment is still a very specialised, time-consuming and hence expensive process in which specialists play a key role. First of all, the creation of an IVE, including object behaviour and user interaction is often done in a low-level programming language. Furthermore, the user's appreciation, when using multiple senses, is not yet fully understood and difficult to predict. Prototype implementations and user experiments are hence more often than not the solution to assess a candidate solution, requiring each solution to be implemented, evaluated and possibly re-implemented.

The VR-DeMo project (Virtual Reality: Conceptual Descriptions and Models for the Realisation of Virtual Environments, IWT 030248) aims to simplify and shorten the development cycle by specifying part of the application using high-level models instead of implementing all aspects by means of programming code. This Model Based User Interface Develop- ment (MBUID) approach allows the developer to easily create, evaluate and adapt VE applications, with a focus on the exploration of interaction techniques. The entire VR-DeMo approach focusses both on the high-level description of the virtual world (scene), as well as the interaction. In this paper, we will only focus on the latter part. For the creation of the scene, we refer the interested reader to [Bille et al., 2004] and [Pellens et al., 2007].

Most existing model-based processes start with some kind of task model, evolving to the final user interface using an incremental approach. Typically, an initial model is automatically transformed to the next model using a given set of mapping rules and algorithms, or by manual adaptation of the designer. When applying this approach, known from formbased user interfaces, directly into the domain of IVE, it turns out that it lacks the ability to describe more advanced and multimodal interaction. A MBUID process that can be applied for the design of an IVE application should therefore be able to describe the UI widgets, as well as the interaction techniques for direct manipulation supporting multimodal input and output (such as speech, gestures and haptics).

MBUID, however, will only have additional value over traditional user interface development when powerful tool support is available. In this paper, we 
describe the capabilities of a tool, called 'CoGenIVE', and its assessment. In the next section we shortly describe the main steps in the VR-DeMo process. Sections 3 through 6 explain how CoGenIVE supports this process. This tool is then assessed in section 7 by describing some practical case studies. We end this paper by comparing our approach to existing related work and we subsequently formulate our conclusions and future research directions.

\section{The VR-DeMo Process}

The design of an IVE application using the VRDeMo approach is a tool-supported process as depicted in figure 1. Before focussing on the toolsupport itself, we shortly explain the main steps in this process. In the next sections, each step is explained in detail, as well as how it is supported by CoGenIVE.

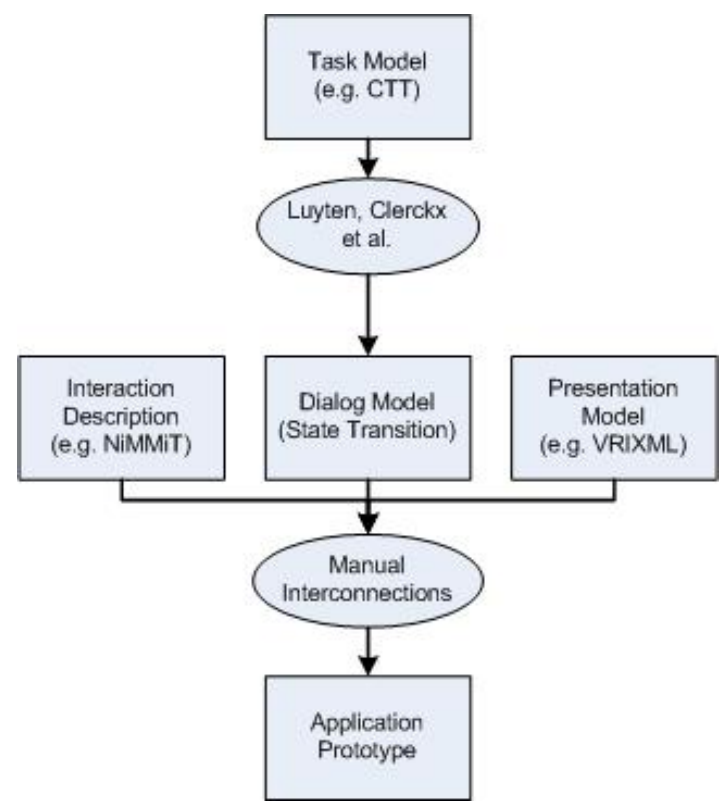

Figure 1: Schematic Overview of the VR-DeMo Process

The process may start from a task-model, describing the possible tasks and their mutual relations. This may contain both tasks performed by the user as well as by the system. From the task-model, a dialog model is derived. As will be explained later in this paper, the first step may be optional so that the designer directly starts by creating a dialog model.

To provide the information for the user interaction, the dialog model is annotated with a presentation model, describing the UI widgets, and an interaction description. The interconnection of the presentation model and the interaction description with the dialog model is a manual process, in which the designer has to indicate which events correspond to a given task.

After annotating the dialog model, an application prototype is built that can be executed immediately. The prototype also contains the application code and some metadata containing the contents of the models. If necessary, a programming specialist can tweak the code. The last step can be considered as an iterative process, which means that the interaction description model, the presentation model, and the final annotation of the dialog model, can be altered, while all changes afterwards are preserved.

\section{Dialog Model}

Although the dialog model forms the center of the CoGenIVE, the VR-DeMo approach leaves to freedom to import a task model. We have chosen not to include a task model editor into CoGenIVE as good editors already exist [Mori et al., 2002].

\subsection{Defining the States}

In CoGenIVE, the dialog model is represented as a state chart, which can be created manually or by importing a task model. Each state represents the tasks that are currently enabled and hence can be performed. For instance, when the user has chosen to manipulate a given object (and thus is in a given 'state' of the application), he can only move or rotate an object, and is for instance unable to create a new object.

The dialog model can be created manually by dragging the states on the canvas and assigning a name to them. The tasks that are enabled for a particular state are assigned as described in section 3.2. Alternatively, the designer may start by creating a task model. For this model, we have chosen to use the ConcurTaskTree (CTT) notation [Paternò, 2000], as this notation provides the required flexibility and allows to make use of temporal relationships between the different tasks. For the creation of a CTT, we propose to use the ConcurTaskTree environment (CTTE). CoGenIVE can transform this model into a dialog model, using the algorithm of Clerckx et al. [Clerckx et al., 2004a]. This transformation groups all tasks that can be executed at a particular moment into an Enabled Task Set (ETS) [Paternò, 2000]. These different ETSs correspond to the states in the dialog model of CoGenIVE.

An example of a dialog model is shown in figure 2. Each rectangle represents a state, while the arrows represent the state transitions. The assignment of tasks and events with a state is explained in section 3.2. 


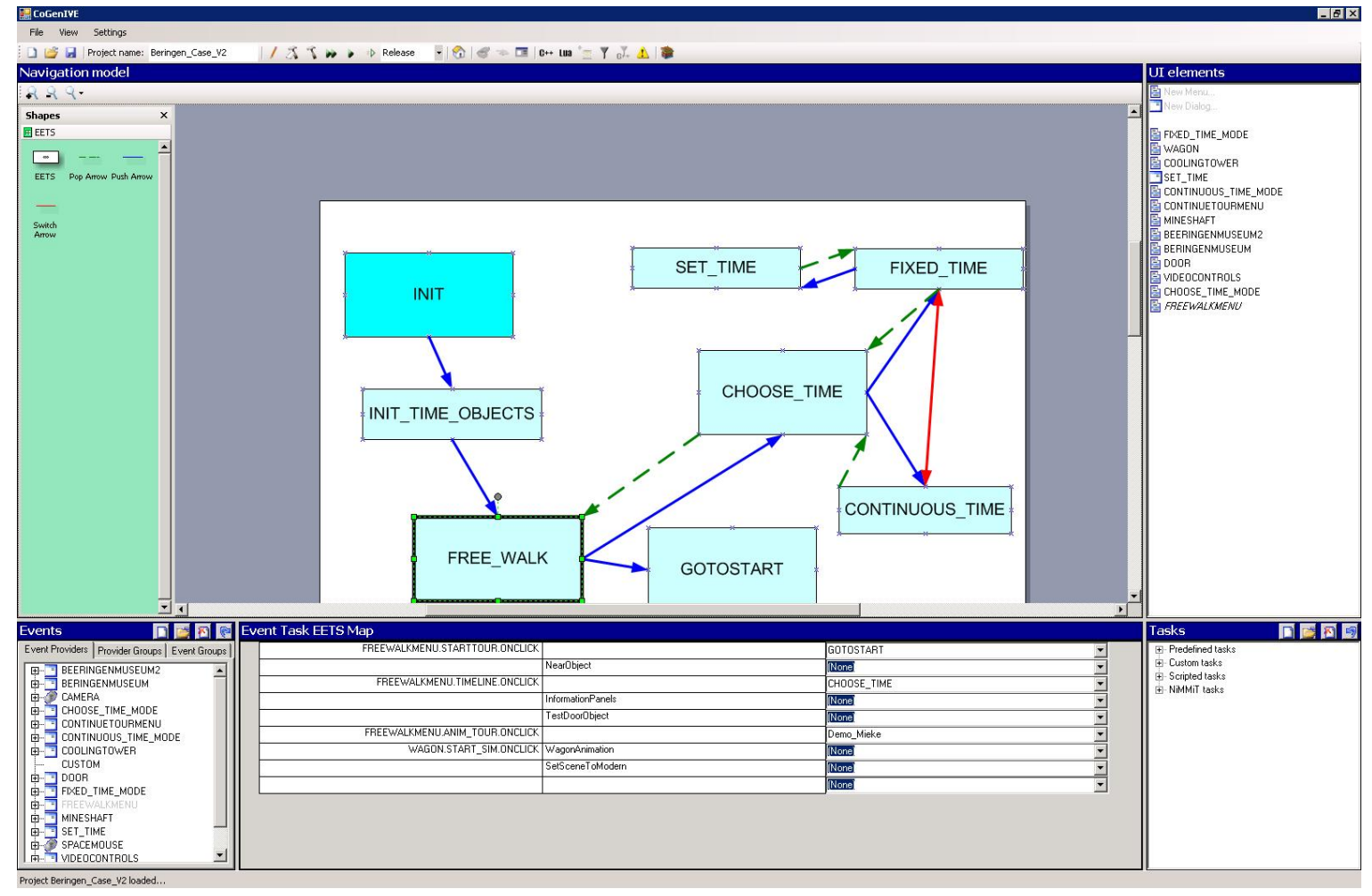

Figure 2: Designing the Dialog Model

\subsection{Handling Input}

Interactive Virtual Environments strongly rely on the user input, which obviously means that we have to define how the user may interact with the system. The next step hence is to define the events that will trigger a task in the current state for execution. Events are generated by event providers, which roughly correspond to the user's input sources, such as a tracker, a speech recognition system (defined by a grammar), a gesture recogniser, or a classic menu or dialog. The event providers and their possible events are listed in the bottom left pane called 'Events'(figure 2), and their aim is to trigger the tasks in the application.

As a first step in the process, we have to define at least one input device as event provider, e.g. a tracker. To add a device, we can choose the type of device we want to connect, as different devices have different properties (number of buttons, force feedback, degrees of freedom, ...). Based upon the selected device the relevant events will appear in the tree. Because we use VRPN [Taylor II et al., 2001] to make abstraction of the concrete device, the setup can be changed at will, later on.

The bottom right pane ('Tasks'), contains a task list. Tasks may be predefined by the system, they can be custom-made for the project using a scripting lan- guage or $\mathrm{C}++$, or they may be an interaction description (as we will explain in section 5). The tasks are finally associated to a state using the window pane in the middle between the events and the tasks. By selecting a state from the dialog model and then dragging an event from the event list, and a task from the task list into the same line we define that the particular event triggers the given task (obviously within the current application state).

Before finishing the dialog model, the designer may need to define a presentation model or interaction description model, respectively to define user interface elements such as menus, or complex user interaction tasks, as will be explained explained in the next sections.

\section{Presentation Model}

In many MBUID approaches, the presentation model describes in an abstract ${ }^{1}$ way how the user interface must look like. From our previous work [Coninx et al., 1997]

\footnotetext{
${ }^{1}$ Abstract in this context means that the model does not take into account features such as the exact placement of a widget or how it exactly looks like on a given platform
} 
[Raymaekers and Coninx, 2001], we have learned that hybrid 2D/3D user interface elements, such as 2D menus or dialogs, positioned in $3 \mathrm{D}$, are effective in virtual environments. In order to avoid having to hard-code these interaction elements, we have created VRIXML, an XML-based User Interface Description Language (UIDL), suited for 2D/3D hybrid menus [Cuppens et al., 2004].

The current version of VRIXML has been extended in order to realise a cooperation between the VRIXML presentation model and the interaction description model. For instance, VRIXML now supports more events, as well as the possibility to attach simple scripts to those event. Those scripts are executed immediately, without the need to handle them in the underlying models. This is especially suitable for simple actions within the dialog, such as the enabling or disabling of certain parts.

The design of the presentation model is integrated in CoGenIVE by choosing a user interface (UI) element from a list (as can be seen in the top right window pane of figure 2). The chosen menu or dialog can then be edited by simple drag-and-drop, and filling out the requested properties (figure 3 ). Typically, each UI element and each item must have a name, defining the final event that will be fired when activated. The presentation model in CoGenIVE is designed using a visualisation which abstracts from the appearance of the elements in the final VE application. Indeed, dependent on the rendering engine and/or the platform, menus and dialogs may have a slightly different appearance.

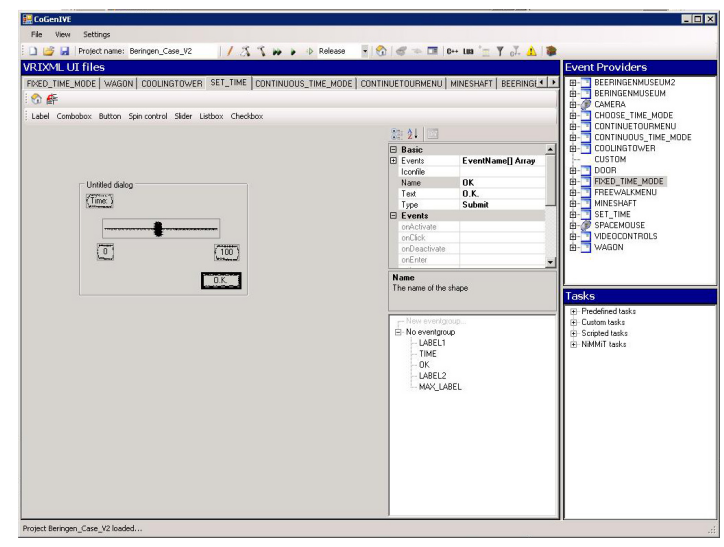

Figure 3: Designing the Presentation Model

The presentation model is serialised as a set of VRIXML files describing the user interface elements and their events. The events generated by the user interface are added to the event list (fig. 2). The name of each UI element appears in the top right list ('UI Elements'). Here it can be assigned to an Enabled Task Set as a default UI element by simply dragging it to the dialog model.

While our presentation model corresponds to presentation models in common MBUID approaches, this is not sufficient to fully describe user interaction in virtual environments. An enriched interaction description, as discussed in the next section, overcomes this lack of expressive power.

\section{Interaction Description}

As most traditional MBUID approaches lack the support for multimodal interaction, we have developed NiMMiT, Notation For MultiModal Interaction Techniques. NiMMiT is developed to describe interaction techniques at a much higher level than by writing code. An interaction technique can be seen a complex ensemble of multimodal information that is merged and applied in order to execute a compound task which consists of several sub-tasks. A good example may be 'touching an object to push it away'. NiMMiT is a graphical notation, inheriting the formalism of a state-chart in order to describe the (multimodal) interaction within the virtual environment. Furthermore, it also supports dataflow which is important in the user interaction, as well. A more detailed description of NiMMiT can be found in [Vanacken et al., 2006] and [Boeck et al., 2007]. We shortly describe the most important primitives of NiMMiT. An example of a NiMMiT diagram can be seen in figure 4.

NiMMiT is basically a state chart, in which a state (represented as a circle) represents the possible events the user can provide and to which the application listens. Besides states, NiMMiT contains the following structures:

- As described in section 3.2, an event is generated by an action a user can perform, such as moving a pointing device, speaking a command, clicking a button, etc. When an event or a combination of events has been occurred, the particular arrow points to a task-chain (big rectangles) that is to be executed.

- A task-chain is a linear succession of tasks that are executed one after the other.

- A task (smaller rectangle in a task-chain) is set of actions defined to 'reach a goal'. A task may be moving an object or calculating collision between objects.

NiMMiT also supports data-flow between different tasks. Labels (high level variables) are used to save output from a task (output ports are depicted as small squares at the bottom right of the task symbol), or to provide input to a task (input ports are depicted at the top-left of a task) 


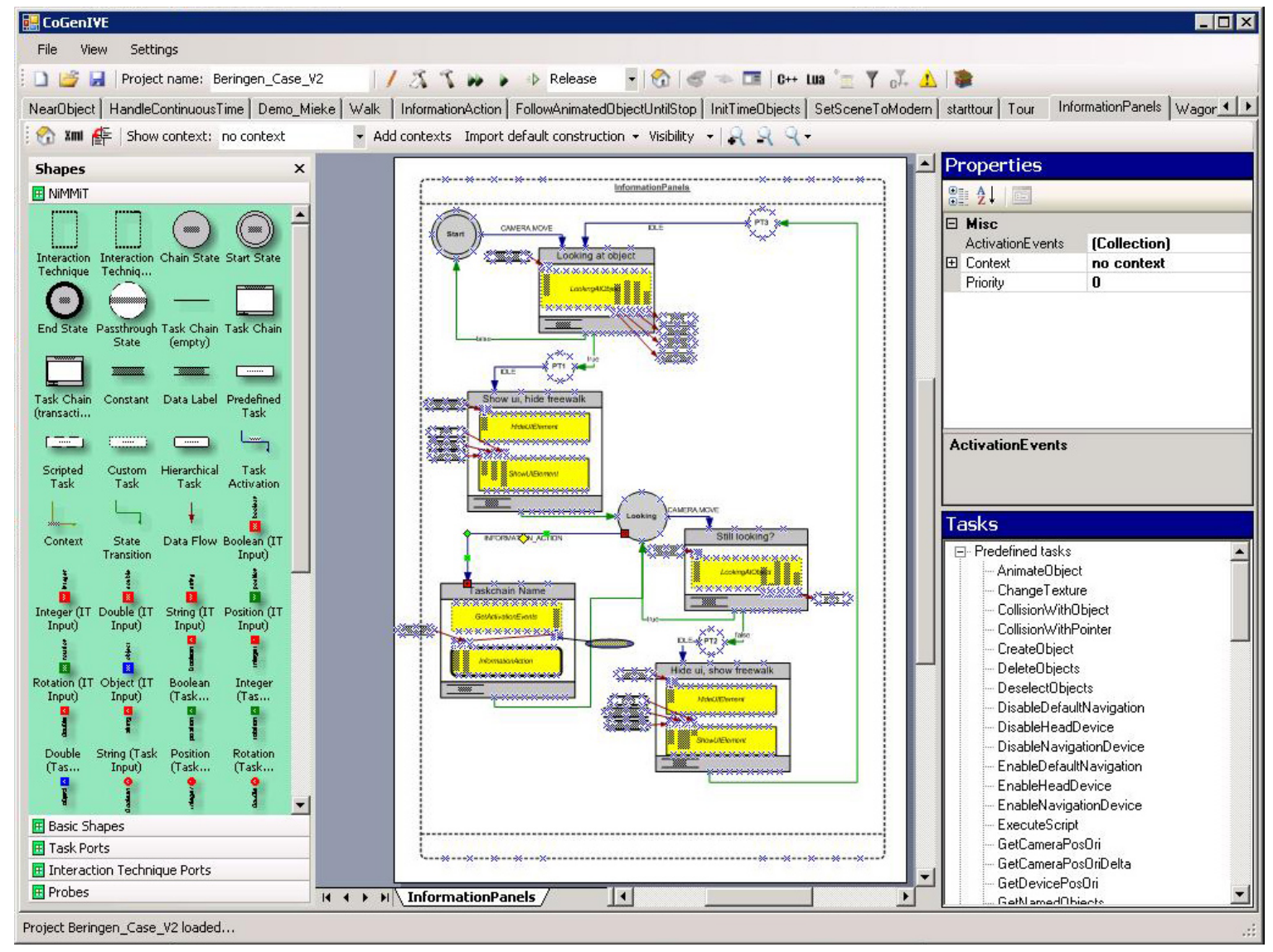

Figure 4: Designing the Interaction Model using the NiMMiT notation

Tasks are mostly predefined, such as selecting an object, calculating collision, etc. so that the user can easily pick them from a list. For specialised actions, however, custom tasks can be written.

When a task-chain is finished, a state-transition occurs (light arrow) bringing the interaction into a new state, responding to another set of events.

In order to support an easy evaluation of the designed diagrams, NiMMiT also defines 'probes', 'filters' and 'listeners'(not depicted in figure 4), primitives that support easy measuring of user performance and evaluating a proposed interaction technique [Coninx et al., 2006].

A NiMMiT diagram is created using the NiMMiT Editor and stored in XML. The XML-file is loaded by the NiMMiT engine and interpreted and executed at run-time responding to the relevant events and executing the desired tasks.

In order to describe an interaction technique using NiMMiT (figure 4) the editor allows to (re)use the events defined by the event providers and UI elements. The tasks in a NiMMiT diagram can be chosen from the tasks list. Moreover, the editor performs several checks and asserts that NiMMiT diagrams are correct. For instance, the editor allows that a 'constant value' can appear at several places in the diagram while it's value is automatically kept up to date. In the same way labels automatically get a data type dependent on the type of the output port they are connected to, and connections of any type have other visual representations when they are not properly connected.

\section{Application Prototype}

Finally, when the dialog model is annotated by the presentation and interaction model and connection has been made between events and tasks, the application can be generated. The application is ready to be run directly from within CoGenIVE, but as the result of this step is also a Visual Studio Project file with the code files (containing the instantiation of all tasks, devices, and the code of the custom tasks), a programming specialist can start tweaking the code within the designated areas. 
It may be stressed here that the final steps in this process (creating the dialog model, presentation model, interaction model, and possibly altering the generated code) may be iterated on, which means that changes in one model or in the hand-written code may be preserved in case another model is adapted. This makes the VR-DeMo approach especially suitable for creating and evaluating prototypes.

In order to evaluate CoGenIVE, we created some practical applications, as described in the next section. These examples illustrate how several prototypes may be proposed to a customer, in order to search for the most satisfying solution in a particular case.

\section{Practical use of CoGenIVE}

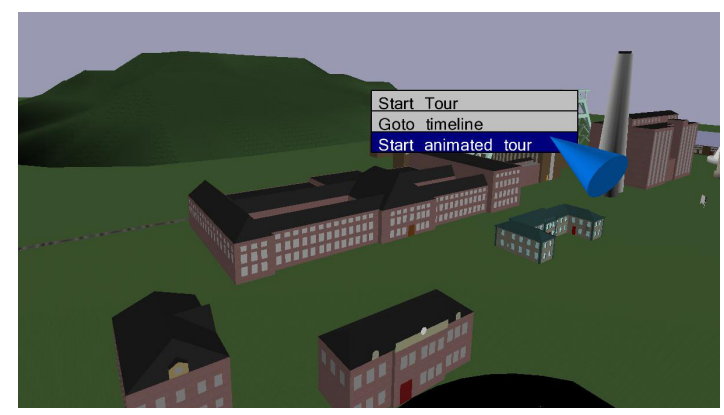

Figure 5: The Virtual Cole Mine Museum

When designing several case studies, we experienced CoGenIVE as an helpful tool, stimulating iterative development and gradual fine-tuning of the interaction.

A first application created with CoGenIVE, is a demonstrator for a cole mine museum (fig 5). The application offers a 3D virtual reproduction of the entire cole mine site, allowing the user to freely explore the site or follow a guided tour, at which the visitor is guided, but still can look around, as he or she is moving their head. Extra information can be requested upon some interesting buildings or items, and the application also contains some animations, illustrating for instance the coal flow in the mine. The application also has a feature to show videos at certain places in the scene, showing movies about the life in the mine, as well as some QuickTime VR scenes giving a view on the interior of the buildings.

As the project leader of the coal mine museum came up with the idea, but did not exactly knew the possibilities of a IVE in a museum environment, a first prototype with the features of a first brainstorm was built. The features included some basic navigation and extra information menus when approaching certain objects. In a second iteration we came to the cur- rent application prototype (including several animations, QuickTime VR, etc.) which now can be used to show the possibilities of such an application to the executives. The time spent for the creation of the entire application, excluding the scene, was about 10 person days.

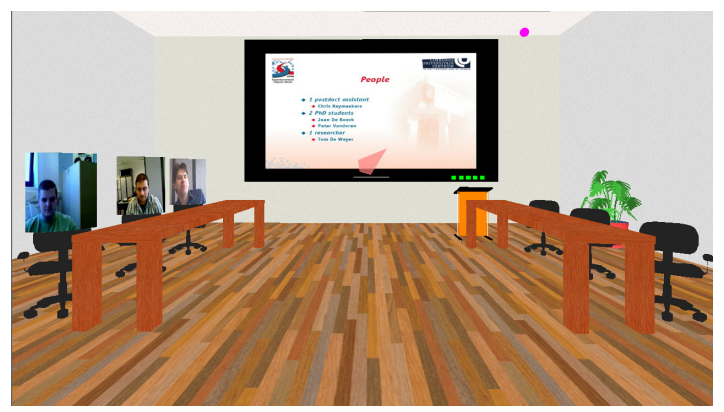

Figure 6: The 3D Teleconferencing Application

A similar approach was applicable for an application prototype for a $3 \mathrm{D}$ teleconferencing application. The project leader wanted to explore the possibilities for a 3D interface supporting teleconferencing. Starting point was to produce an attractive, but easy to use interface, with some (but not concretely specified) features such as participants who can start a presentation. A first prototype has been built and shown to the project leader. In subsequent iterations, some features were added, and others removed. In general this resulted in an interface in which features could be activated with the least amount of clicks possible.

The time required to come to the first version was about 12 persons days, including the time for the interfacing with the existing teleconferencing library ${ }^{2}$ To come to the final prototype, as shown in figure 6 , we required another 4 person days. These time calculations include the modeling of the application, but exclude the creation of the scene.

The examples above illustrate the possibility of our tool-supported process to easily create application prototypes at a reasonable time. We have also used CoGenIVE in a context where user interaction was less important, or at least less of a question. In the latter application, a 3D driving simulator had to be created. Although the accent in this project laid on the simulation aspects, which had to be manually coded, CoGenIVE played a key role in creating the application and designing the overall application structure, breaking-up the simulation process in several smaller building blocks which all could be implemented by 'custom tasks', written in $\mathrm{C}++$. In this kind of situations, the benefit of the tool is not only quickly gener-

\footnotetext{
${ }^{2} \mathrm{We}$ interfaced with ANDROME's Intellivic SDK (www.intellivic.com), writing the interface code as "custom tasks' in our model based process.
} 
ating an initial version. The tools also aids in flexible extensions while keeping a structured design.

\section{Related Work}

MBUID has been largely investigated in the context of standard form-based user interfaces [Vanderdonckt, 2005] Especially the need for a flexible design in current state of the art user interfaces supporting multiple devices [Mori et al., 2004], contextual adaptations, or distribution and migration of UIs [Clerckx et al., 2004b] indicate the possibilities of a MBUID approach. This topic, however, is fairly new in the domain of interactive virtual environments. In this section, we will shortly describe how the VR-DeMo process and CoGenIVE are related to other work.

Although the need for an easier development of a user interface in a virtual environment is existent, not so much related research activities can be found. Some toolkits, such as VRJuggler [Bierbaum et al., 2001] offer facilities to a programmer in order to build a VE application much faster. The applications, however still have to written in programming code. Willans et al. [Willans J.S. and S.P., 2000] propose a methodology that separates the process of designing object behaviour from the process of building a virtual world. They use existing techniques such as flownets to describe the behaviour. Similarly, Tanriverdi describes how VRID (Virtual Reality Interface Design) [Tanriverdi and Jacob, 2001] is used to divide the development process in a high-level and a low-level phase. The first phase helps designers to conceptually design the interface without implementation specific terminology. The Low-level phase helps to represent design specifications in an implementation oriented terminology. Finally, a commercial tool to easily develop virtual environments is VirTools [Virtools inc, 2007]. It allows a user to define the object behaviour and user interaction using a graphical notation. Although most approaches have their contribution towards the facilitation of the development cycle, most of them focus directly on programming issues, rather than on the design and analysis. This leaves a significant gap and several open issues to apply MDUID in practice in an IVE [De Boeck et al., 2006a].

In its general application of form-based user interfaces, several popular UIDLs exist that can be used to describe the presentation model. UIML (User Interface Markup Language) [Abrams and Phanouriou, 1999] is a widely used standard. It is an XML-based meta-language that permits a declarative and device independent description of a user interface. Because of its generality, it is possible to use UIML in stead of languages such as VoiceXML, or WML. But clearly, UIML's generality implies that its complexity is a main drawback. UsiXML (User Interface Extensible Markup Language) [Vanderdonckt et al., 2004] is a description language that can be used to describe a user interface at different levels (going from abstract to concrete user interfaces). UsiXML already has been applied in a variety of domains, recently including VR [Gonzalez et al., 2006]. More information on how VRIXML relates to other UIMLs can be found in [Cuppens et al., 2004].

For the description of user interaction, mainly two families of notations do exist: state-driven notations and data-driven notations. For the Interaction Description Model, we used NiMMiT, which inherits the formalisms of a state chart [Harel, 1987], but adopting some principles of dataflow as well. Popular State-driven notations are Petri-nets [Palanque and Bastide, 1994], coloured petri-nets [Jensen, 1994] or ICO [Navarre et al., 2005]. Data driven notations include InTML [Figueroa et al., 2002] or UML activity diagrams [Ambler, 2004]. For a more comprehensive overview of the related work on this topic, we refer the interested reader to [Vanacken et al., 2006], [Boeck et al., 2007] and [De Boeck et al., 2006b].

\section{Conclusions}

In this paper we elaborated on a tool 'CoGenIVE', supporting the VR-DeMo process, a model based design process to create VE applications. We showed how CoGenIVE supports the different steps in the process, such as creating the dialog model, the presentation and the interaction model. The approach described above facilitates the development of an IVE in general, but especially creates an environment in which alternative features within the 3D world can be easily tried. This has been successfully applied in some practical cases we described in this paper: the virtual coal mine museum and the $3 \mathrm{D}$ video conference application. But CoGenIVE has also been applied in a context where 'prototyping' was much less a requirement, creating a car simulator.

Based upon our experience we can conclude that, once the VR-DeMo process is known, CoGenIVE offers the designer a useful tool to create VE applications. 


\section{Acknowledgements}

Part of the research at the Expertise Centre for Digital Media is funded by the ERDF (European Regional Development Fund), the Flemish Government and the Flemish Interdisciplinary institute for Broadband Technology (IBBT). The VR-DeMo project (IWT 030248) is directly funded by the IWT, a Flemish subsidy organization.

The authors also want to thank Erwin Cuppens, Tom De Weyer, Tim Tutenel and Lode Vanacken for their valuable contributions to CoGenIVE. We also want to thank the partners of the VR-DeMo user committee involved in the practical demonstrator applications.

\section{REFERENCES}

[Abrams and Phanouriou, 1999] Abrams, M. and Phanouriou, C. (1999). Uiml: An xml language for building device-independent user interfaces. In XML '99, Philadelphia, USA.

[Ambler, 2004] Ambler, S. (2004). Object Primer, The Agile Model-Driven Development with UML 2.0. Cambridge University Press.

[Bierbaum et al., 2001] Bierbaum, A., Just, C., Hartling, P., Meinert, K., Baker, A., and Cruz-Neira, C. (2001). VR juggler: A virtual platform for virtual reality application development. In Proceedings of IEEE Virtual Reality Conference 2001, Yokohama - Japan.

[Bille et al., 2004] Bille, W., Pellens, B., Kleinermann, F., and De Troyer, O. (2004). Intelligent modelling of virtual worlds using domain ontologies. In Proceedings of the Workshop of Intelligent Computing (WIC), held in conjunction with the MICAI 2004 conference, pages 272 -279, Mexico City, Mexico.

[Boeck et al., 2007] Boeck, J. D., Vanacken, D., Raymaekers, C., and Coninx, K. (2007). High-level modeling of multimodal interaction techniques using nimmit. Journal of Virtual Reality and Broadcasting, 4(2). urn:nbn: de:0009-6-11615.

[Clerckx et al., 2004a] Clerckx, T., Luyten, K., and Coninx, K. (2004a). Dynamo-AID: A design process and a runtime architecture for dynamic model-based user interface development. In 9th IFIP Working Conf. on Engineering for Human-Computer Interaction jointly with 11th Int. Workshop on Design, Speci-fication, and Verification of Interactive Systems EHCI-DSVIS 2004, pages 77-95, Hamburg, Germany. Springer-Verlag.

[Clerckx et al., 2004b] Clerckx, T., Luyten, K., and Coninx, K. (2004b). Dynamo-AID: a design process and a runtime architecture for dynamic model-based user interface development. In Proceedings of EHCIDSVIS'04, pages 142-160, Tremsbüttle Castle, Hamburg, Germany.
[Coninx et al., 2006] Coninx, K., Cuppens, E., De Boeck, J., and Raymaekers, C. (2006). Integrating support for usability evaluation into high level interaction descriptions with NiMMiT. In Proceedings of 13th International Workshop on Design, Specification and Verification of Interactive Systems (DSVIS'06), volume 4385, Dublin, Ireland.

[Coninx et al., 1997] Coninx, K., Van Reeth, F., and Flerackers, E. (1997). A hybrid 2D/3D user interface for immersive object modeling. In Proceedings of Computer Graphics International '97, pages 47-55, Hasselt and Diepenbeek, BE.

[Cuppens et al., 2004] Cuppens, E., Raymaekers, C., and Coninx, K. (2004). VRIXML: A user interface description language for virtual environments. In Developing User Interfaces with XML: Advances on User Interface Description Languages, pages 111-117, Gallipoli, Italy.

[De Boeck et al., 2006a] De Boeck, J., Gonzalez Calleros, J. M., Coninx, K., and Vanderdonckt, J. (2006a). Open issues for the development of $3 \mathrm{~d}$ multimodal applications from an MDE perspective. In MDDAUI workshop 2006, Genova, Italy.

[De Boeck et al., 2006b] De Boeck, J., Raymaekers, C., and Coninx, K. (2006b). Comparing NiMMiT and datadriven notations for describing multimodal interaction. In Tamodia 2006, Diepenbeek, Belgium.

[Figueroa et al., 2002] Figueroa, P., Green, M., and Hoover, H. J. (2002). InTml: A description language for VR applications. In Proceedings of Web3D'02, pages 53-58, Arizona, USA.

[Gonzalez et al., 2006] Gonzalez, J., Vanderdonckt, J., and Arteaga, J. (2006). A Method for Developing 3D User Interfaces of Information Systems, chapter 7, pages 85100. Proc. of 6th Int. Conf. on Computer-Aided Design of User Interfaces CADUI2́206. Springer-Verlag, Bucharest, Berlin.

[Harel, 1987] Harel, D. (1987). Statecharts: A visual formalism for complex systems. In Science of Computer Programming, volume 8, pages 321-274.

[Jensen, 1994] Jensen, K. (1994). An introduction to the theoretical aspects of coloured petri nets. In W.-P. de Roever, G. Rozenberg (eds.): A Decade of Concurrency, Lecture Notes in Computer Science, volume 803, pages 230-272. Springer-Verlag.

[Mori et al., 2002] Mori, G., Paternò, F., and Santoro, C. (2002). CTTE: support for developing and analyzing task models for interactive system design. IEEE Transactions on Software Engineering, 28(8):797-813.

[Mori et al., 2004] Mori, G., Paternò, F., and Santoro, C. (2004). Design and development of multidevice user interfaces through multiple logical descriptions. IEEE Transactions On Software Engineering, 30(8):1 - 14.

[Navarre et al., 2005] Navarre, D., Palanque, P., Bastide, R., Schyn, A., Winckler, M., Nedel, L., and Freitas, C. (2005). A formal description of multimodal interaction techniques for immersive virtual reality applications. In Proceedings of Tenth IFIP TC13 International Conference on Human-Computer Interaction, Rome, IT. 
[Palanque and Bastide, 1994] Palanque, P. and Bastide, R. (1994). Petri net based design of user-driven interfaces using the interactive cooperative objects formalism. In Interactive Systems: Design, Specification, and Verification, pages 383-400. Springer-Verlag.

[Paternò, 2000] Paternò, F. (2000). Model-Based Design and Evaluation of Interactive Applications. SpringerVerlag.

[Pellens et al., 2007] Pellens, B., De Troyer, O., Kleinermann, F., and Bille, W. (2007). Conceptual modeling of behavior in a virtual environment. Special issue: International Journal of Product and Development, 4(6):626645.

[Raymaekers and Coninx, 2001] Raymaekers, C. and Coninx, K. (2001). Menu interactions in a desktop haptic environment. In Proceedings of Eurohaptics 2001, pages 49-53, Birmingham, UK.

[Tanriverdi and Jacob, 2001] Tanriverdi, V. and Jacob, R. (2001). VRID a design model and methodology for developing virtual reality interfaces. In Proceedings of ACM Symposium on Virtual Reality Software and Technology, Alberta - Canada.

[Taylor II et al., 2001] Taylor II, R., Hudson, T., Seeger, A., Weber, H., Juliano, J., and Helser., A. (2001). VRPN: A device-independent, network-transparent vr peripheral system. In In Proceedings of the ACM, pages 55-61.
[Vanacken et al., 2006] Vanacken, D., De Boeck, J., Raymaekers, C., and Coninx, K. (2006). NiMMiT: A notation for modeling multimodal interaction techniques. In Proceedings of the International Conference on Computer Graphics Theory and Applications (GRAPPO6), Setbal, Portugal.

[Vanderdonckt et al., 2004] Vanderdonckt, J., Limbourg, Q., Michotte, B., Bouillon, L., Trevisan, D., and Florins, M. (2004). Usixml: a user interface description language for specifying multimodal user interfaces. In Proceedings of W3C Workshop on Multimodal Interaction WMI'2004, pages 35-42, Sophia Antipolis.

[Vanderdonckt, 2005] Vanderdonckt, J. A. M. (2005). Compliant environment for developing user interfaces of information systems. In Proc. of 17th Conf. on Advanced Information Systems Engineering CAiSE'05, pages 1631, Porto, Portugal.

[Virtools inc, 2007] Virtools inc (August 2007). Virtools Dev. http://www.virtools.com.

[Willans J.S. and S.P., 2000] Willans J.S., H. M. and S.P., S. (2000). Implementing virtual environment object behavior from a specification. pages $87-97$. 